\title{
Variational approach to thermal masses in compactified models
}

\author{
Daniele Dominici $^{a}$ and Itzhak Roditi ${ }^{b}$ \\ ${ }^{a}$ Dipartimento di Fisica e Astronomia Università di Firenze and INFN — Sezione di Firenze, \\ Via G. Sansone 1, 50019 Sesto Fiorentino, Italy \\ ${ }^{b}$ Centro Brasileiro de Pesquisas Físicas - CBPF/MCT, \\ Rua Dr. Xavier Sigaud 150, 22290-180, Rio de Janeiro, RJ, Brazil \\ E-mail: dominici@fi.infn.it, roditi@cbpf.br
}

ABSTRACT: We investigate by means of a variational approach the effective potential of a $5 D \mathrm{U}(1)$ scalar model at finite temperature and compactified on $S^{1}$ and $S^{1} / Z_{2}$ as well as the corresponding $4 D$ model obtained through a trivial dimensional reduction. We are particularly interested in the behavior of the thermal masses of the scalar field with respect to the Wilson line phase and the results obtained are compared with those coming from a one-loop effective potential calculation. We also explore the nature of the phase transition.

KEYwords: Field Theories in Higher Dimensions, Effective field theories

ARXIV EPRINT: 1507.01365 


\section{Contents}

1 Introduction 1

2 The reduced model 2

3 Compactified 5D scalar QED 5

\section{Introduction}

Studies on field theories at finite temperature have had a regain of interest particularly to investigate the electroweak transition and baryogenesis both in the Standard Model (SM) as effective low energy theory in 4 dimensions and in its extensions in five dimensions [15]. These new approaches have some of their theoretical roots in finite temperature field theory [6-9]. Considering topological aspects of a thermal formalism, it is realized that the prescription results in a scheme of compactification in time of the $T=0$ theory. That is, the Matsubara results are equivalent to a path-integral calculated on $R^{D-1} \times S^{1}$, where $S^{1}$ is a circle of circumference $\beta=1 / T$. In the case of an extra dimension corresponding to $S^{1} / \mathbf{Z}_{2}$ orbifold one should also take into account the parity projection [10]. As a consequence, the Matsubara prescription can be thought, in a generalized way, as a mechanism to deal simultaneously with spatial constraints and thermal effects in a field theory model. This concept has been developed for the Matsubara formalism by considering $R^{D-d} \times S^{l_{1}} \times$ $S^{l_{2}} \cdots \times S^{l_{d}}$, with $l_{1}$ corresponding to inverse temperature and $l_{2}, \cdots l_{d}$ corresponding to compactification of $d-1$ spatial dimensions. Then the Feynman rules are modified by introducing a generalized Matsubara prescription, performing the following multiple replacements (compactification of a $d$-dimensional subspace) [11],

$$
\int \frac{d k_{1}}{2 \pi} \rightarrow \frac{1}{\beta} \sum_{n_{1}=-\infty}^{+\infty}, \quad \int \frac{d k_{i}}{2 \pi} \rightarrow \frac{1}{L_{i}} \sum_{n_{i}=-\infty}^{+\infty} ; \quad k_{1} \rightarrow \frac{2 n_{1} \pi}{\beta} \quad k_{i} \rightarrow \frac{2 n_{i} \pi}{L_{i}}, \quad i=2,3 \ldots, d-1
$$

where $L_{i}, \quad i=2,3 \ldots, d-1$ are the sizes of the compactified spatial dimensions.

The ideas above have found new and interesting applications in the context of field theories with extra spatial dimensions, where the Higgs field is identified as the zero mode of the fifth component of the gauge field, as for instance in [10, 12-27]. Models of this type are sometimes called models with gauge-Higgs unification. Perhaps these theories provide an interesting framework for physics beyond the SM.

In particular, there has been a resurgence of interest in these ideas, as a new way to investigate the electroweak transition and baryogenesis. The electroweak phase transition, in 5-dimensional finite-temperature field theory with a compactified extra dimension, has been investigated in [2]. These authors compare theirs results with those obtained with the 
SM at one-loop approximation. The conclusion is for a first-order transition with a strength inversely proportional to the Higgs mass, using the estimated value of the Higgs mass, of $M_{H}<M_{W} / 2$. However, these authors also claim that when fermions in the uncompactified 5 -D space are introduced, more realistic values of the Higgs mass are obtained. In this case the first-order phase transition becomes weaker. Another interesting result of [2] is that up to temperatures of the order of $T \approx 1 / L$, reliable (low order) perturbative calculations lead to reasonable results. It has been recently noticed that a $\mathrm{SO}(5) \times \mathrm{U}(1)$ gauge Higgs unification in the Randall Sundrum metric can reproduce the Higgs mass at $125 \mathrm{GeV}$ for three fermion spinorial representations and shown that the thermal phase transition at one loop is first order but very weak so baryogenesis could not occur [5].

From a physical and phenomenological point of view, an interest in theories with extra compactified dimensions at the inverse $\mathrm{TeV}$ scale arose in connection with the new LHC experiments.

In order to go beyond one-loop approximation, self-consistent approaches have been also considered. One example is the Cornwall-Jackiw-Tomboulis effective action for composite operators that has been generalized to finite temperature [28-30]. Here we follow an alternative route, which consists in developing another, non perturbative, variational technique, the Gaussian Effective Potential (GEP) [31, 32], for both finite temperature and compactified spatial dimensions applying it as a tool to investigate effective field theories.

The aim of this paper is to study, by using the GEP, the thermal masses of two models. One being the reduced model, at finite temperature, obtained from neglecting all massive Kaluza Klein modes for a 5-dimensional U(1) model, thus trivially reducing it to a 4-dimensional model. The other being the full 5-dimensional U(1) model, at finite temperature, compactified over $S^{1}$ and over the $S^{1} / \mathbf{Z}_{2}$ orbifold. Some of our results confirm the perturbative results of [2].

In section 2 we consider first the GEP for the reduced model where a truncation of the Kaluza Klein expansion has been performed, so that only the first KK mode is retained. In section 3 we consider the full 5D scalar electrodynamics, deriving the GEP at finite temperature and calculating the Higgs thermal mass. We also discuss the structure of the phase transition by looking into the high temperature limit.

\section{The reduced model}

In this section we review the reduced model which provides a simple exercise for studying the effective potential for the five dimensional scalar electrodynamics by truncating the Kaluza Klein tower and considering only a first scalar lower mode. Let us consider the following lagrangian:

$$
\mathcal{L}=\left(D_{\mu} \varphi\right)^{\dagger}\left(D^{\mu} \varphi\right)+\frac{1}{2}\left(\partial_{\mu} s\right)^{2}-g^{2} s^{2} \varphi^{\dagger} \varphi-\frac{\lambda}{4}\left(\varphi^{\dagger} \varphi\right)^{2},
$$

which corresponds to the reduced $4 D$ model.

We introduce real fields $\hat{\phi}_{1}, \hat{\phi}_{2}$,

$$
\varphi=\frac{1}{\sqrt{2}}\left(\hat{\phi}_{1}+i \hat{\phi}_{2}\right)
$$


and perform a shift on the scalar field $s$,

$$
s(x)=\hat{s}(x)+s_{c}
$$

which allows to interpret $s_{c}$ as a constant background field.

In the canonically quantized version of the Gaussian effective potential (GEP) [31, 32] the quantum free fields $\hat{\phi}$ and $\hat{s}$, respectively of masses $\Omega$ and $\Delta$, are expanded in the form,

$$
\hat{\phi}=\int \frac{d^{3} k}{(2 \pi)^{3} 2 \omega_{k}}\left[a_{\Omega}(k) e^{-i k x}+\text { h.c. }\right]
$$

with $\omega_{k}^{2}=\vec{k}^{2}+\Omega^{2}$ and

$$
\hat{s}=\int \frac{d^{3} k}{(2 \pi)^{3} 2 \omega_{k}}\left[a_{\Delta}(k) e^{-i k x}+\text { h.c. }\right]
$$

with $\omega_{k}^{2}=\vec{k}^{2}+\Delta^{2}$. Standard commutation relations for creation and annihilation operators are assumed for $a_{\Sigma}, a_{\Sigma}^{\dagger}$ :

$$
\left[a_{\Sigma}(k), a_{\Sigma}\left(k^{\prime}\right)^{\dagger}\right]=2 \omega_{k}(2 \pi)^{3} \delta^{3}\left(k-k^{\prime}\right)
$$

where $\Sigma$ stands for either $\Omega$ or $\Delta$. In the Gaussian effective potential approach, the effective potential is evaluated as the expectation value of the Hamiltonian

$$
V_{G}(\alpha, \Omega, \Delta)=<0|\mathcal{H}| 0>
$$

where the vacuum $|0\rangle$ is annihilated by $a_{\Omega}(k)$ and $a_{\Delta}(k)$ and $\mathcal{H}$ is the total Hamiltonian associated to the lagrangian density (2.1). Also we have redefined $s_{c}=\alpha / g R, 2 \pi R$ being the length of the fifth compactified dimension . One can then minimize $V_{G}$ with respect to the parameters $\Sigma$ obtaining the associated gap equations which provide the values of the parameters that, when replaced in $V_{G}$, gives the Gaussian effective potential $\bar{V}_{G}$

An equivalent calculation may be performed by use of the first order $\delta$-expansion [33, 34], which amounts to split the Lagrangian $\mathcal{L}$ into a sum of a "free field" $\mathcal{L}_{0}$ term and a $\mathcal{L}_{\delta}$ term containing the interactions, such that when $\delta=1$ the original Lagrangian is recovered. In the next section it will be convenient to cast our calculations into this approach. In both cases, the result for the reduced model is:

$$
\begin{aligned}
V_{G}=2 I_{1}(\Omega)-\Omega^{2} & I_{0}(\Omega)+I_{1}(\Delta)-\frac{1}{2} \Delta^{2} I_{0}(\Delta) \\
& +g^{2} I_{0}(\Delta) I_{0}(\Omega)+\frac{\lambda}{2} I_{0}^{2}(\Omega)+\frac{\alpha^{2}}{R^{2}} I_{0}(\Omega) .
\end{aligned}
$$

where

$$
I_{0}(\Sigma)=\int \frac{d^{3} p}{(2 \pi)^{3}} \frac{1}{2 \omega_{p}} \quad I_{1}(\Sigma)=\int \frac{d^{3} p}{(2 \pi)^{3}} \frac{1}{2} \omega_{p}
$$

with $\omega_{p}=\sqrt{\mathbf{p}^{2}+\Sigma^{2}}$. The $I_{0}$ and $I_{1}$ integrals are equivalent to the covariant form (in the Euclidean and for the $I_{1}$ case up to an infinite constant)

$$
I_{0}(\Sigma)=\int \frac{d^{4} p}{(2 \pi)^{4}} \frac{1}{p^{2}+\Sigma^{2}} \quad I_{1}(\Sigma)=\frac{1}{2} \int \frac{d^{4} p}{(2 \pi)^{4}} \log \left[p^{2}+\Sigma^{2}\right]
$$


The gap equations are:

$$
\begin{aligned}
& \Omega^{2}=\frac{\alpha^{2}}{R^{2}}+\lambda I_{0}(\Omega)+g^{2} I_{0}(\Delta), \\
& \Delta^{2}=2 g^{2} I_{0}(\Omega) .
\end{aligned}
$$

where use has been done of the identity

$$
\frac{d}{d \Sigma} I_{1}(\Sigma)=\Sigma I_{0}(\Sigma)
$$

Replacing the values of $\Omega$ and $\Delta$ from eq. (2.11) into eq. (2.8) gives the 'optimized' Gaussian effective potential, $\bar{V}_{G}$. The divergent integrals $I_{0}, I_{1}$ are evaluated using dimensional regularization with the minimal subtraction scheme $(\overline{\mathrm{MS}})$ prescription. In particular one has (in the Euclidean and using their covariant form)

$$
\begin{aligned}
& I_{0}(\Sigma)=\int \frac{d^{4} p}{(2 \pi)^{4}} \frac{1}{p^{2}+\Sigma^{2}}=\frac{\Sigma^{2}}{(4 \pi)^{2}}\left(\log \frac{\Sigma^{2}}{\mu^{2}}-1\right) \\
& I_{1}(\Sigma)=\frac{1}{2} \int \frac{d^{4} p}{(2 \pi)^{4}} \log \left(p^{2}+\Sigma^{2}\right)=\frac{\Sigma^{4}}{64 \pi^{2}}\left(\log \frac{\Sigma^{2}}{\mu^{2}}-\frac{3}{2}\right)
\end{aligned}
$$

where $\mu$ is a regularization scale. Using this regularization prescription, eq. (2.12) is still valid and therefore the form of the gap equation is the same as eq. (2.11).

The finite temperature result can be obtained by replacing the $I$ integrals by their finite temperature version $I^{F T}$, as described in $[35,36]$. For $I_{1}^{F T}$, one has

$$
I_{1}^{F T}=I_{1}+I_{1}^{\beta}
$$

where the parameter $\beta$ indicates, as usual, the inverse of the temperature $T$ and

$$
\begin{aligned}
I_{1}^{\beta}(\Sigma) & =\frac{1}{\beta} \int \frac{d^{3} p}{(2 \pi)^{3}} \log \left(1-e^{-\beta w_{p}}\right) \\
& =\frac{1}{\beta^{4}}\left[-\frac{\pi^{2}}{90}+\frac{(\beta \Omega)^{2}}{24}-\frac{(\beta \Omega)^{3}}{12 \pi}-\frac{(\beta \Omega)^{4}}{64 \pi^{2}}\left(\log \beta^{2} \Omega^{2}-c\right)\right],
\end{aligned}
$$

with $c=3 / 2+2(\log 4 \pi-\gamma) \sim 5.41$. It is important to observe that the structure of the Gaussian effective potential is maintained as eq. (2.12) is also valid for the finite temperature integral $I_{1}^{F T}$. With these substitutions one is then led to a finite temperature Gaussian effective potential $\bar{V}_{G}^{F T}$.

Having obtained the optimized finite temperature Gaussian effective potential we can calculate the Wilson line thermal mass of the field $\alpha$, i.e.,

$$
M^{2}(T, \alpha)=\mathcal{N} \frac{\partial^{2} \bar{V}_{G}^{F T}}{\partial \alpha^{2}}
$$

where $\mathcal{N}$ is a suitable normalization factor.

As we are dealing with an effective theory, a series of approximations are in order and so, following [2] we shall assume that $g$ is negligible. In that case, we can consider 


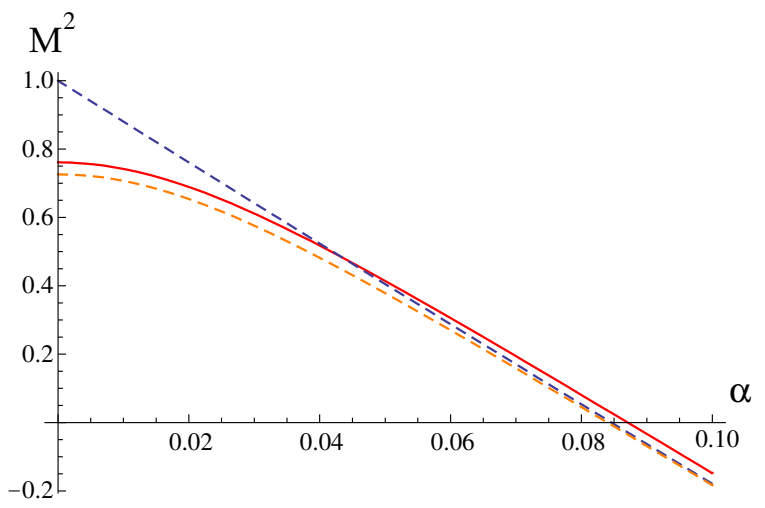

Figure 1. Squared thermal mass of the field $\alpha$ in the reduced model from the GEP (continuous line), one-loop effective potential (upper dashed line), improved one-loop (lower dashed line) for $L T=1, \lambda=1, \mu=1 / R$.

that, from eq. (2.11), $\Delta=0$, and within the dimensional regularization scheme one can set scale-independent integrals as $I_{0}(0)$ or $I_{1}(0)$ equal to zero. So we are led to a simpler situation where only the equation for $\Omega$ needs to be satisfied. Assuming that $L T=1, \lambda=1$ and $\mu=1 / R$, the scale above which one considers that the effective theory breaks down, we plot $M^{2}(T, \alpha)$, in figure 1 , for $\bar{V}_{G}^{F T}$ (red line) and compare it with the results for the improved one-loop effective potential (red dashed line) and the "maive one-loop effective potential (upper dashed line) taken from [2], which, in our notation, is simply proportional to the integral $I_{1}^{F T}(\Omega)$ for a value $\Omega=\alpha / R$. The normalization factor $\mathcal{N}$ is chosen such as to make equal to 1 the thermal mass calculated from the naïve one-loop effective potential when $\alpha=0$. The main result is that the thermal mass calculated with GEP is substantially in agreement (slightly higher) with the prediction of the improved one-loop method.

\section{Compactified 5D scalar QED}

Let us now consider 5D U(1) scalar electrodynamics, with the 5th dimension compactified on a circle $S^{1}$ of length $L=2 \pi R$, defined in a Euclidean space by the action

$$
S_{E}=\int_{-\pi R}^{\pi R} d y \int d^{4} x\left[\frac{1}{4} F_{M N} F_{M N}+\left(D_{M} \varphi\right)^{\dagger}\left(D_{M} \varphi\right)+\frac{\lambda_{5}}{4}\left(\varphi^{\dagger} \varphi\right)^{2}+\mathcal{L}_{g f}\right]
$$

with $\mathcal{L}_{g f}$ being the gauge fixing term and

$$
D_{M} \varphi=\partial_{M} \varphi+i g_{5} A_{M} \varphi
$$

where $\varphi$ satisfies the boundary condition $\varphi(2 \pi R)=\varphi(0)$ and capital letters indicate $5 D$ indices. Notice that the change from Minkowskian to Euclidean space corresponds to $V_{M} T^{M} \rightarrow-V_{M} T_{M}$, therefore after a redefinition of sign in the action. We adopt the convention that capital Roman letter label indices running from 1 to 5, while Greek letters are used for indices which run from 1 to 4 . We are interested in considering the effective potential for the vacuum solution for the 5 th component of the field $A_{M}$. Therefore we 
will consider the $A_{M}$ field as a background field with only $A_{5}\left(\mathbf{x}, x_{5}\right) \neq 0$, where we have used the notation $\mathbf{x} \equiv\left(x_{1}, \cdots, x_{4}\right)$ for the coordinates of the position vector in the noncompactified subspace. Here, as mentioned, it will be more convenient to introduce the Gaussian effective potential as the first order in the $\delta$-expansion [33, 34].

Introducing the real components of $\varphi$,

$$
\varphi=\frac{1}{\sqrt{2}}\left(\hat{\phi}_{1}+i \hat{\phi}_{2}\right)
$$

the part of the Lagrangian quadratic in $\hat{\phi}_{1,2}$ can be rewritten as

$$
\frac{1}{2} \Phi^{T} D^{-1} \Phi
$$

with $\Phi=\left(\hat{\phi}_{1}, \hat{\phi}_{2}\right)$

$$
D^{-1}=\left(\begin{array}{cc}
-\partial_{M}^{2}+g_{5}^{2} A_{5}^{2} & 2 g_{5} A_{M} \partial_{M} \\
-2 g_{5} A_{M} \partial_{M} & -\partial_{M}^{2}+g_{5}^{2} A_{5}^{2}
\end{array}\right)
$$

Then we shift the field $A_{5}$ by a constant background field $\mathcal{A}_{5}$ getting,

$$
\hat{A}_{5}\left(\mathbf{x}, x_{5}\right)=A_{5}\left(\mathbf{x}, x_{5}\right)-\mathcal{A}_{5}
$$

and we split the lagrangian as

$$
\mathcal{L}=\left(\mathcal{L}_{0}+\mathcal{L}_{\text {int }}\right)_{\delta=1}
$$

where $\mathcal{L}_{0}$ is the sum of the quadratic lagrangian for $\hat{\phi}_{1,2}$, the electromagnetic field terms and the terms containing the variational parameter $\Omega$ :

$$
\mathcal{L}_{0}=\frac{1}{2}\left[\hat{\Phi} \hat{\mathcal{D}} \hat{\Phi}+\left(\partial_{\mu} \hat{A}_{5}\right)^{2}\right]
$$

with

$$
\hat{\mathcal{D}}^{-1}=\left(\begin{array}{cc}
-\partial_{M}^{2}+\frac{\alpha^{2}}{R^{2}}+\Omega^{2} & 2 \frac{\alpha}{R} \partial_{5} \\
-2 \frac{\alpha}{R} \partial_{5} & -\partial_{M}^{2}+\frac{\alpha^{2}}{R^{2}}+\Omega^{2}
\end{array}\right),
$$

where we have used $g_{5} \mathcal{A}_{5}=\alpha / R$ as well as $\lambda_{5}=L \lambda$.

The interaction Lagrangian is then:

$$
\begin{aligned}
\mathcal{L}_{\mathrm{int}}=\delta\left\{\frac{1}{2}\left[\frac{\alpha^{2}}{R^{2}}-\Omega^{2}\right]\left(\hat{\phi}_{1}^{2}+\hat{\phi}_{2}^{2}\right)+\frac{L \lambda}{16} \hat{\phi}_{1}^{4}+\frac{L \lambda}{16} \hat{\phi}_{2}^{4}+\frac{L \lambda}{8} \hat{\phi}_{1}^{2} \hat{\phi}_{2}^{2}\right. \\
\left.+\frac{1}{2} g_{5}^{2} \hat{A}_{5}^{2}\left(\hat{\phi}_{1}^{2}+\hat{\phi}_{2}^{2}\right)-\Delta^{2} \hat{A}_{5}^{2}\right\} .
\end{aligned}
$$

In eq. (3.10), for simplicity, we kept only the quadratic terms that survive after the functional integrations.

Introducing sources for the fields, the generating functional is written as:

$$
Z[J, j]=\int \mathrm{D}\left[\hat{\phi}_{1}, \hat{\phi}_{2}, \hat{A}_{5}\right] \exp \left(-\int_{-\pi R}^{\pi R} d x_{5} \int d^{4} x\left\{\mathcal{L}_{0}+\mathcal{L}_{\text {int }}\right\}+\langle J . \Phi\rangle+\left\langle j . A_{5}\left(\mathbf{x}, x_{5}\right)\right\rangle\right),
$$


and the effective action is obtained by the Legendre transformation

$$
\Gamma[\alpha]=\ln Z[j]-\left\langle j . A_{5}\right\rangle,
$$

where the brackets \langle\rangle are a shorthand notation for the integral $\int d x_{5} d^{4} x$.

One can expand the action, eq. (3.12), to first order in $\delta$ by the usual functional techniques getting,

$$
\begin{aligned}
\Gamma_{\mathrm{GEP}}=\frac{1}{2} \operatorname{Tr} \ln \left(\mathcal{D}^{-1}\right) & +\frac{1}{2} \operatorname{Tr} \ln \left(G_{5}^{-1}\right) \\
+ & <\mathcal{L}\left(\frac{\delta}{\delta(J, j)}\right)>\left.\exp \left[\frac{1}{2}\left(<J^{T} \cdot \mathcal{D} . J>+<j G_{5} j>\right)\right]\right|_{J, j=0}
\end{aligned}
$$

where, again, the brackets are a short notation for the integrations and the summations over the compactified variables, $\mathcal{L}\left(\frac{\delta}{\delta(J, j)}\right)$ means that we have replaced the fields $\hat{\phi}_{1}, \hat{\phi}_{2}$ and $\hat{A}_{5}$ by their functional derivatives in $J_{1}, J_{2}$ and $j$. The inverse of $\hat{\mathcal{D}}$ in the momentum representation is given by

$$
\hat{\mathcal{D}}^{-1}=\left(\begin{array}{cc}
p_{M}^{2}+\frac{\alpha^{2}}{R^{2}}+\Omega^{2} & 2 i \frac{\alpha}{R} p_{5} \\
-2 i \frac{\alpha}{R} p_{5} & p_{M}^{2}+\frac{\alpha^{2}}{R^{2}}+\Omega^{2}
\end{array}\right) .
$$

The result, after the calculation of the traces and omitting the terms in $\mathrm{g}$ (as mentioned at the end of the previous section, $g$ is considered negligible), reduces to

$$
V_{G}=\tilde{I}_{1}(\Omega)-\frac{1}{2} \Omega^{2} \tilde{I}_{0}(\Omega)+\frac{1}{2} \frac{\alpha^{2}}{R^{2}} \tilde{I}_{0}(\Omega)+\frac{L \lambda}{2} \tilde{I}_{0}^{2}(\Omega),
$$

where the integrals $I_{0}$ and $I_{1}$, prior to compactification are given by:

$$
\begin{aligned}
\tilde{I}_{0}(\Omega) & =\left[\int \frac{d^{5} p}{(2 \pi)^{5}} \frac{1}{p^{2}+\left(p_{5}+\frac{\alpha}{R}\right)^{2}+\Omega^{2}}+\int \frac{d^{5} p}{(2 \pi)^{5}} \frac{1}{p^{2}+\left(p_{5}-\frac{\alpha}{R}\right)^{2}+\Omega^{2}}\right] \\
& =\sum_{q= \pm 1} \int \frac{d^{5} p}{(2 \pi)^{5}} \frac{1}{p^{2}+\left(p_{5}+q \frac{\alpha}{R}\right)^{2}+\Omega^{2}}
\end{aligned}
$$

and

$$
\begin{aligned}
\tilde{I}_{1}(\Omega) & =\frac{1}{2} \int \frac{d^{5} p}{(2 \pi)^{5}} \log \left[\left(p^{2}+\left(p_{5}+\frac{\alpha}{R}\right)^{2}+\Omega^{2}\right)\left(p^{2}+\left(p_{5}-\frac{\alpha}{R}\right)^{2}+\Omega^{2}\right)\right] \\
& =\sum_{q= \pm 1} \frac{1}{2} \int \frac{d^{5} p}{(2 \pi)^{5}} \log \left[\left(p^{2}+\left(p_{5}+q \frac{\alpha}{R}\right)^{2}+\Omega^{2}\right)\right] .
\end{aligned}
$$

The gap equation, obtained by minimizing the potential $V_{G}$ with respect to the parameter $\Omega$, in this case is:

$$
\Omega^{2}=\frac{\alpha^{2}}{R^{2}}+2 L \lambda \tilde{I}_{0}(\Omega) .
$$

As before replacing the value of $\Omega$ eq. (3.18) into eq. (3.15) gives the value of Gaussian effective potential, $\bar{V}_{G}$, now for the compactified scalar QED. Formally, when the value $\bar{\Omega}$ gives a minimum, it is possible to replace eq. (3.18) in eq. (3.15), such that:

$$
\bar{V}_{G}=\tilde{I}_{1}(\bar{\Omega})-\frac{L \lambda}{2} \tilde{I}_{0}^{2}(\bar{\Omega}) .
$$


In order to introduce simultaneously the compactification of the 5th dimension over $S^{1}$ and a finite temperature, one has to perform the replacements,

$$
\frac{d^{5} p}{(2 \pi)^{5}} \rightarrow \frac{T}{L} \sum_{k, n=-\infty}^{+\infty} \int \frac{d^{3} p}{(2 \pi)^{3}} ; \quad p_{5} \rightarrow \frac{2 \pi k}{L}, \quad p_{4} \rightarrow 2 \pi n T .
$$

Remembering that $L=2 \pi R$, we finally get,

$$
\tilde{I}_{0}^{F T}(\Omega)=\frac{T}{4 \pi R} \sum_{q= \pm 1} \sum_{k, n=-\infty}^{+\infty} \int \frac{d^{3} p}{(2 \pi)^{3}} \frac{1}{\left[\vec{p}^{2}+(2 \pi n T)^{2}+\Omega^{2}+\frac{(k+q \alpha)^{2}}{R^{2}}\right]}
$$

and

$$
\tilde{I}_{1}^{F T}(\Omega)=\frac{T}{8 \pi R} \sum_{q= \pm 1} \sum_{k, n=-\infty}^{+\infty} \int \frac{d^{3} p}{(2 \pi)^{3}} \log \left[\vec{p}^{2}+(2 \pi n T)^{2}+\Omega^{2}+\frac{(k+q \alpha)^{2}}{R^{2}}\right]
$$

In eqs. (3.20) to (3.22) the sums over $n$ and $k$ refer respectively to the Matsubara and compactification modes. The motivation to go through this procedure, in order to obtain eq. (3.22), is that this expression is much easier to manipulate by means of the Poisson summation formula,

$$
\sum_{\ell=-\infty}^{+\infty} e^{-\pi t(\ell+a)^{2}}=\frac{1}{\sqrt{t}} \sum_{\kappa=-\infty}^{+\infty} e^{-\frac{\pi \kappa^{2}}{t}} e^{2 i \pi \kappa a}=\frac{1}{\sqrt{t}}\left[1+2 \sum_{\kappa=1}^{+\infty} e^{-\frac{\pi \kappa^{2}}{t}} \cos (2 \pi \kappa a)\right]
$$

together with the identity

$$
\operatorname{tr} \log M=-\int_{0}^{\infty} \frac{d t}{t} \operatorname{tr} e^{-t M}
$$

Using eqs. (3.23), (3.24) and the representation of Bessel functions of the third kind, $K_{\nu}$,

$$
2(A / B)^{\frac{\nu}{2}} K_{\nu}(2 \sqrt{A B})=\int_{0}^{\infty} d x x^{-\nu-1} e^{-(A / x)-B x},
$$

the integrals, $\tilde{I}_{0}^{F T}$ and $\tilde{I}_{1}^{F T}$, subtracting the divergent term corresponding to the zero mode, which however does not depend on $\alpha$ [2], can be written as:

$$
\tilde{I}_{0}^{F T}=\frac{T^{3}}{2(2 \pi)^{5 / 2}} \sum_{q= \pm 1} \sum_{\kappa=1}^{+\infty} \sum_{\ell=-\infty}^{+\infty} \frac{\cos (2 \pi \kappa q \alpha)}{\left[\ell^{2}+(L T \kappa)^{2}\right]^{\frac{3}{2}}} B_{\frac{3}{2}}\left(\frac{\Omega}{T} \sqrt{\ell^{2}+(L T \kappa)^{2}}\right)
$$

and

$$
\tilde{I}_{1}^{F T}=-\frac{2 T^{5}}{(2 \pi)^{5 / 2}} \sum_{q= \pm 1} \sum_{\kappa=1}^{+\infty} \sum_{\ell=-\infty}^{+\infty} \frac{\cos (2 \pi \kappa q \alpha)}{\left[\ell^{2}+(L T \kappa)^{2}\right]^{\frac{5}{2}}} B_{\frac{5}{2}}\left(\frac{\Omega}{T} \sqrt{\ell^{2}+(L T \kappa)^{2}}\right)
$$

where $B_{\nu}(z)=z^{\nu} K_{\nu}(z)$.

In the calculations above we have performed the Poisson resummation over both the Matsubara $(\ell)$ and compactification $(\kappa)$ modes. Equivalently, it is possible to use this 
procedure only over the Matsubara modes or only over the compactification ones. The results are:

$$
\tilde{I}_{0}^{F T}=\frac{T}{8(2 \pi)^{2} L^{2}} \sum_{q= \pm 1} \sum_{\kappa=1}^{+\infty} \sum_{\ell=-\infty}^{+\infty} \frac{\cos (2 \pi \kappa q \alpha)}{\kappa^{2}} B_{1}\left(L \kappa \sqrt{\Omega^{2}+(2 \pi T \ell)^{2}}\right),
$$

and

$$
\tilde{I}_{1}^{F T}=-\frac{2 T}{(2 \pi)^{2} L^{4}} \sum_{q= \pm 1} \sum_{\kappa=1}^{+\infty} \sum_{\ell=-\infty}^{+\infty} \frac{\cos (2 \pi \kappa q \alpha)}{\kappa^{4}} B_{2}\left(L \kappa \sqrt{\Omega^{2}+(2 \pi T \ell)^{2}}\right),
$$

for the compactification modes, or

$$
\begin{aligned}
\tilde{I}_{0}^{F T}= & \frac{1}{(2 \pi)^{5 / 2} L^{3}} \sum_{q= \pm 1} \sum_{\kappa=1}^{+\infty} \frac{\cos (2 \pi \kappa q \alpha)}{\kappa^{3}} B_{3 / 2}(L \Omega \kappa) \\
& +\frac{T^{2}}{(2 \pi)^{2} L} \sum_{q= \pm 1} \sum_{\kappa=-\infty}^{+\infty} \sum_{\ell=1}^{+\infty} \frac{1}{\ell^{2}} B_{1}\left(\frac{\ell}{T} \sqrt{\Omega^{2}+\frac{1}{R^{2}}(\kappa+q \alpha)^{2}}\right)
\end{aligned}
$$

and

$$
\begin{aligned}
\tilde{I}_{1}^{F T}= & -\frac{2}{(2 \pi)^{5 / 2} L^{5}} \sum_{q= \pm 1} \sum_{\kappa=1}^{+\infty} \frac{\cos (2 \pi \kappa q \alpha)}{\kappa^{5}} B_{5 / 2}(L \Omega \kappa) \\
& -\frac{2 T^{4}}{(2 \pi)^{2} L} \sum_{q= \pm 1} \sum_{\kappa=-\infty}^{+\infty} \sum_{\ell=1}^{+\infty} \frac{1}{\ell^{4}} B_{2}\left(\frac{\ell}{T} \sqrt{\Omega^{2}+\frac{1}{R^{2}}(\kappa+q \alpha)^{2}}\right)
\end{aligned}
$$

for the Matsubara modes.

We have then laid the setup for the calculation of the thermal mass of the field $\alpha$. In figure 2 we compare the results of the squared thermal mass $M^{2}$ obtained with the GEP (red continuous line), for $L T=1$ and $\lambda=1$, with the improved one-loop effective potential [2]. The variational calculation result is larger than the improved one-loop for small values of $\alpha$ and smaller for larger value. In fact, the variational calculation interpolates the naif one-loop and improved results for the region of small values of $\alpha$ and is more sensitive than the perturbative approaches in the larger values of $\alpha$ region.

It is also interesting to notice that, as in [2] we can obtain an indication of the order of the phase transition. Using the expressions of eqs. (3.28) and (3.29) for the end point $\Omega=0$ and dropping all the modes, but the zero mode, in the Matsubara expansion we arrive at:

$$
\tilde{I}_{0}^{F T}=\frac{T}{8(2 \pi)^{2} L^{2}} \sum_{q= \pm 1} \sum_{\kappa=1}^{+\infty} \frac{\cos (2 \pi \kappa q \alpha)}{\kappa^{2}}
$$

and

$$
\tilde{I}_{1}^{F T}=-\frac{T}{\pi^{2} L^{4}} \sum_{q= \pm 1} \sum_{\kappa=1}^{+\infty} \frac{\cos (2 \pi \kappa q \alpha)}{\kappa^{4}},
$$

where we have also used $\lim _{\xi \rightarrow 0} B_{\nu}(\xi)=2^{\nu-1} \Gamma(\nu)$. 


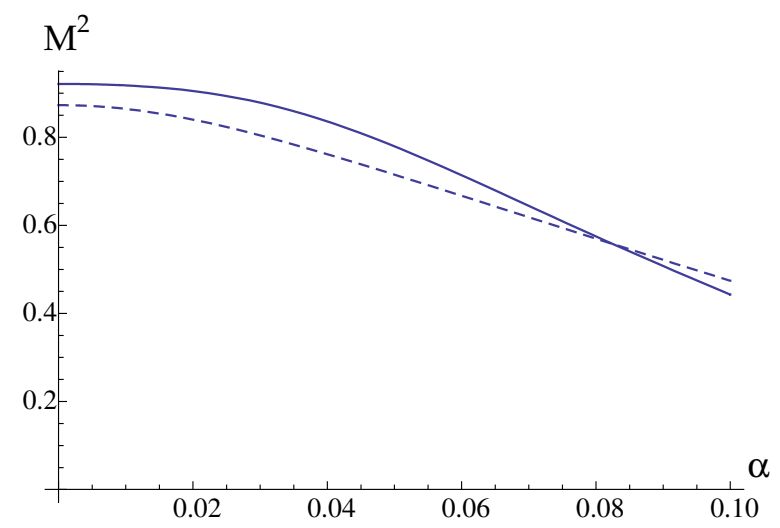

Figure 2. Squared thermal mass of the field $\alpha$ in the 5D model using the GEP (continuous line) and the improved one-loop (dashed line) for $L T=1$ and $\lambda=1$.

The cosinus function in eqs. (3.32) and (3.33) lead to ill-defined, rapidly oscillating series. Nevertheless, there is a solution in the framework of the zeta-function regularization. This procedure has already been used in [2] and it is a crucial step to these authors conclude for a first-order electroweak transition. As it is well-known, the zeta function can be analytically extended to the whole complex plane, having only one pole at $z=1$. This analytical extension with a strictly negative even argument vanishes: $\zeta(-2 n)=0$ for integer $n \geq 1$. Accordingly, we expand the cosinus functions above in a power series of $\alpha$ and using $\sum_{\kappa=1}^{+\infty} \kappa^{2 n}=\zeta(-2 n)=0$ for all positive integers $n \geq 1$ it is possible to get, for definite values of $q$ (we use $\zeta(0)=-1 / 2)$ ):

$$
\tilde{I}_{0}^{F T}=\frac{T}{32 L^{2}}\left(\frac{1}{6}-q \alpha+(q \alpha)^{2}\right)
$$

and

$$
\tilde{I}_{1}^{F T}=-\frac{\pi^{2} T}{3 L^{4}}\left(\frac{1}{30}-(q \alpha)^{2}+2(q \alpha)^{3}-(q \alpha)^{4}\right)
$$

Now, the reasoning for doing these manipulations is that the $\Omega=0$ endpoint corresponds to an infrared limit [2,31], also one can see that in eqs. (3.28) and (3.29) when the temperature, $T$, is very large and for any non null $\ell$, the generalized Bessel function $B_{\lambda}$ approaches zero and the extremum values for the integrals occurs at the $\Omega=0$ end point. Under these conditions the potential will be given by replacing eqs. (3.34) and (3.35) in eq. (3.15) taking the endpoint $\Omega=0$.

In the $S^{1}$ compactification case we keep all degrees of freedom and the odd powers of $\alpha$ cancel out . One gets, apart from a term independent of $\alpha$, a polynomial of order 4 in $\alpha$ for the potential, which has the form,

$$
V_{G}=\frac{\pi^{2}}{48 L^{5}}\left(A \alpha^{2}+B \alpha^{4}\right)
$$

where the coefficients $A=33 \rho+\lambda \rho^{2} /\left(32 \pi^{2}\right)$ and $B=38 \rho+3 \lambda \rho^{2} /\left(32 \pi^{2}\right)$ are positive and $\rho=L T$. Since we are in the high temperature regime $T L \gg 1$, we expect the system to be 
in the disordered phase. So, for $\lambda$ positive and when we keep all degrees of freedom, the form of eq. (3.36) indicates the system has undergone a second-order phase transition.

The $S^{1} / Z_{2}$ compactification runs in a similar way, with the caveat that one has to project the states over a definite value for the charge $q$ [10], we have to choose only one value of $q$ for the expressions in eqs. (3.34) and (3.35); in this case the odd powers of $\alpha$ do not cancel. That means that an $\alpha^{3}$ term remains in the effective potential. Its form is proportional to $\alpha^{3}\left(35 / 48 \rho+\lambda \rho^{2} /(32 \pi)^{2}\right.$ indicating that for positive values of $\lambda$ the system has undergone a first order phase transition.

\section{Acknowledgments}

I.R. thanks the warm hospitality of the Dipartimento di Fisica Università di Firenze where parts of this work were done. He also acknowledges fruitful discussions as well as crucial suggestions from Adolfo P.C. Malbouisson. The authors acknowledge partial financial support from INFN, Compaq and PRIN contract 2010YJ2NYW.

Open Access. This article is distributed under the terms of the Creative Commons Attribution License (CC-BY 4.0), which permits any use, distribution and reproduction in any medium, provided the original author(s) and source are credited.

\section{References}

[1] C. Grojean, G. Servant and J.D. Wells, First-order electroweak phase transition in the standard model with a low cutoff, Phys. Rev. D 71 (2005) 036001 [hep-ph/0407019] [INSPIRE].

[2] G. Panico and M. Serone, The electroweak phase transition on orbifolds with gauge-Higgs unification, JHEP 05 (2005) 024 [hep-ph/0502255] [INSPIRE].

[3] N. Maru and K. Takenaga, Aspects of phase transition in gauge-Higgs unification at finite temperature, Phys. Rev. D 72 (2005) 046003 [hep-th/0505066] [INSPIRE].

[4] C. Delaunay, C. Grojean and J.D. Wells, Dynamics of non-renormalizable electroweak symmetry Breaking, JHEP 04 (2008) 029 [arXiv:0711.2511] [INSPIRE].

[5] H. Hatanaka, Thermal phase transition in the $\mathrm{SO}(5) \times \mathrm{U}(1)$ gauge-Higgs unification with 126 GeV Higgs, arXiv: 1304.5104.

[6] T. Matsubara, A new approach to quantum statistical mechanics, Prog. Theor. Phys. 14 (1955) 351 [INSPIRE].

[7] H. Ezawa, Y. Tomozawa and H. Umezawa, Quantum statistics of fields and multiple production of mesons, Nuovo Cim. 5 (1957) 810 [INSPIRE].

[8] R. Kubo, Statistical mechanical theory of irreversible processes. 1. General theory and simple applications in magnetic and conduction problems, J. Phys. Soc. Jap. 12 (1957) 570 [INSPIRE].

[9] P.C. Martin and J.S. Schwinger, Theory of many particle systems. 1, Phys. Rev. 115 (1959) 1342 [INSPIRE]. 
[10] M. Kubo, C.S. Lim and H. Yamashita, The Hosotani mechanism in bulk gauge theories with an orbifold extra space $S^{1} / Z_{2}$, Mod. Phys. Lett. A 17 (2002) 2249 [hep-ph/0111327] [INSPIRE].

[11] F.C. Khanna, A.P. Malbouisson, J.M. Malbouisson and A.R. Santana, Thermal quantum field theory, World Scientific (2009).

[12] D. Fairlie, Two consistent calculations of the Weinberg angle, J. Phys. G 5 (1979) L55 [INSPIRE].

[13] D.B. Fairlie, Higgs' fields and the determination of the Weinberg angle, Phys. Lett. B 82 (1979) 97 [INSPIRE].

[14] N.S. Manton, A new six-dimensional approach to the Weinberg-Salam model, Nucl. Phys. B 158 (1979) 141 [INSPIRE].

[15] Y. Hosotani, Dynamical mass generation by compact extra dimensions, Phys. Lett. B 126 (1983) 309 [INSPIRE].

[16] Y. Hosotani, Dynamical gauge symmetry breaking as the Casimir effect, Phys. Lett. B 129 (1983) 193 [INSPIRE].

[17] I. Antoniadis, A possible new dimension at a few TeV, Phys. Lett. B 246 (1990) 377 [INSPIRE].

[18] C.-L. Ho and Y. Hosotani, Symmetry breaking by Wilson lines and finite temperature effects, Nucl. Phys. B 345 (1990) 445 [inSPIRE].

[19] G.R. Dvali, S. Randjbar-Daemi and R. Tabbash, The origin of spontaneous symmetry breaking in theories with large extra dimensions, Phys. Rev. D 65 (2002) 064021 [hep-ph/0102307] [INSPIRE].

[20] L.J. Hall, Y. Nomura and D. Tucker-Smith, Gauge Higgs unification in higher dimensions, Nucl. Phys. B 639 (2002) 307 [hep-ph/0107331] [INSPIRE].

[21] N. Arkani-Hamed, A.G. Cohen and H. Georgi, Anomalies on orbifolds, Phys. Lett. B 516 (2001) 395 [hep-th/0103135] [INSPIRE].

[22] G. Burdman and Y. Nomura, Unification of Higgs and gauge fields in five-dimensions, Nucl. Phys. B 656 (2003) 3 [hep-ph/0210257] [INSPIRE].

[23] K. Agashe, R. Contino and A. Pomarol, The minimal composite Higgs model, Nucl. Phys. B 719 (2005) 165 [hep-ph/0412089] [INSPIRE].

[24] M. Serone, The Higgs boson as a gauge field in extra dimensions, AIP Conf. Proc. 794 (2005) 139 [hep-ph/0508019] [INSPIRE].

[25] G. Panico, M. Serone and A. Wulzer, A model of electroweak symmetry breaking from a fifth dimension, Nucl. Phys. B 739 (2006) 186 [hep-ph/0510373] [INSPIRE].

[26] Y. Hosotani, K. Oda, T. Ohnuma and Y. Sakamura, Dynamical electroweak symmetry breaking in $\mathrm{SO}(5) \times \mathbf{U}(1)$ gauge-Higgs unification with top and bottom quarks, Phys. Rev. D 78 (2008) 096002 [Erratum ibid. D 79 (2009) 079902] [arXiv:0806.0480] [INSPIRE].

[27] G. Panico, M. Safari and M. Serone, Simple and realistic composite Higgs models in flat extra dimensions, JHEP 02 (2011) 103 [arXiv:1012.2875] [INSPIRE].

[28] A. Barducci, R. Casalbuoni, D. Dominici, R. Gatto and G. Pettini, Composite operator formalism at finite temperature: application to the $O(N)$ model, Phys. Lett. B 179 (1986) 275 [INSPIRE]. 
[29] G. Amelino-Camelia and S.-Y. Pi, Selfconsistent improvement of the finite temperature effective potential, Phys. Rev. D 47 (1993) 2356 [hep-ph/9211211] [INSPIRE].

[30] G. Smet, T. Vanzielighem, K. Van Acoleyen and H. Verschelde, A 2 loop 2PPI analysis of lambda $\phi^{4}$ at finite temperature, Phys. Rev. D 65 (2002) 045015 [hep-th/0108163] [InSPIRE].

[31] P.M. Stevenson, The gaussian effective potential. 1. Quantum mechanics, Phys. Rev. D 30 (1984) 1712 [INSPIRE].

[32] P.M. Stevenson, The gaussian effective potential. 2. $\lambda \phi^{4}$ field theory, Phys. Rev. D 32 (1985) 1389 [INSPIRE].

[33] A. Okopinska, Nonstandard expansion techniques for the effective potential in $\lambda \phi^{4}$ quantum field theory, Phys. Rev. D 35 (1987) 1835 [INSPIRE].

[34] I. Stancu and P.M. Stevenson, Second order corrections to the gaussian effective potential of $\lambda \phi^{4}$ theory, Phys. Rev. D 42 (1990) 2710 [inSPIRE].

[35] I. Roditi, Scalar fields at finite temperature: the gaussian effective potential approach, Phys. Lett. B 169 (1986) 264 [INSPIRE].

[36] G.A. Hajj and P.M. Stevenson, Finite temperature effects on the gaussian effective potential, Phys. Rev. D 37 (1988) 413 [INSPIRE]. 